\title{
Individual Differences in Spatial Ability: Developing Technologies to Increase Strategy Awareness and Skills
}

\section{Susanne P. Lajoie}

To cite this article: Susanne P. Lajoie (2003) Individual Differences in Spatial Ability: Developing Technologies to Increase Strategy Awareness and Skills, Educational Psychologist, 38:2, 115-125, DOI: 10.1207/S15326985EP3802_6

To link to this article: https://doi.org/10.1207/S15326985EP3802_6

曲 Published online: 08 Jun 2010.

Submit your article to this journal $\llbracket$

Џ Article views: 208

Q View related articles $\longleftarrow$

Citing articles: 19 View citing articles 4 


\title{
Individual Differences in Spatial Ability: Developing Technologies to Increase Strategy Awareness and Skills
}

\author{
Susanne P. Lajoie \\ Department of Educational and Counselling Psychology \\ McGill University
}

\begin{abstract}
Individual differences in spatial ability were explored among educational psychology and engineering students as they related to performance on a real-world spatial task known as the orthographic projection task. As expected, educators performed higher on verbal aptitude tests than engineers, and engineers performed higher on spatial aptitude tests than psychologists. A detailed cognitive task analysis revealed specific spatial strategies for solving the orthographic projection tasks. These detailed strategies were used to design a computerized learning environment called the orthographic projection tutor (OPT). Pre- and posttests of orthographic projections were administered to a treatment group and 2 control groups. The results indicated that spatial processes could be identified and taught to certain individuals. Aptitude process research can lead to prescriptive forms of adaptive technology. The connection between theory and the design of computer-based learning environments is discussed.
\end{abstract}

The articles represented in this issue are dedicated to Richard E. Snow and represent both the theoretical and practical influences he had on the field of educational psychology. From a theoretical perspective, the work on aptitude led to countless empirical studies in the area of aptitude processes. The identification of individual differences in aptitudes led to practical implications for both instruction and assessment. The research presented in this article describes the intersection of aptitude process research and instruction, using technology as a medium for assisting learners to develop their spatial aptitude. One aspect of Snow's legacy has been how his research on individual differences led to adaptive forms of instruction using technology, be they intelligent tutoring systems, microworlds, or computer-based learning environments (for a detailed review, see Corno et al., 2002). This article describes how theories of aptitude and learning connect in the design of a computer-based tutoring system called the orthographic projection tutor (OPT). OPT was designed to assist individuals interpret two-dimensional drawings with regard to the three-dimensional objects they represent. Mechanical engineers and architects use orthographic projection drawings in their building plans.

Requests for reprints should be sent to Susanne P. Lajoie, Department of Educational and Counselling Psychology, 3700 McTavish St., McGill University, Montreal, Quebec, Canada H3A 1Y2. E-mail: lajoie@education.mcgill.ca

\section{COGNITIVE APTITUDE RESEARCH}

Individuals differ in a variety of cognitive aptitudes for learning from instruction, and no one instructional treatment has been found optimal for all kinds of students. It is clear that student aptitude and instructional treatment variables interact (Cronbach \& Snow, 1977; Snow, 1977). However, research on aptitude-treatment interactions (ATIs) has not generated consistent findings that suggest prescriptions for adaptive instruction. On the other hand, information processing analysis, in conjunction with ATI research, can provide diagnostic assessments of processing strengths and weaknesses of individuals on particular tasks and, perhaps, guide instructional design for specific individuals (Snow, 1980, 1989).

There are two major issues considered in this research. The first study examines the relation between established aptitude measures and a spatial task found in engineering, the orthographic projection task. A cognitive task analysis was performed that examined individual differences in solving this task as a basis for instructional design of a computerized tutor, OPT. The second study tests whether OPT can teach individuals strategies for solving this real-world spatial task.

\section{SPATIAL ABILITY}

Spatial abilities form a part of the visual thinking used in everyday life. Common activities, such as maneuvering a car 
along an unfamiliar road or rearranging furniture, require visual thinking as do the more complex tasks of translating blueprints into buildings (McKim, 1980). High spatial ability is related to successful performance in real-world occupations such as engineering and architecture (Cronbach, 1970; Smith, 1964).

Research on spatial aptitude has determined the factors that underlie spatial ability and the constituents of such factors (Cooper, 1975; Glushko \& Cooper, 1978; Lohman, 1979; Pellegrino, Mumaw, \& Shute, 1984; Shepard \& Feng, 1972; Shepard \& Metzler, 1971). Some researchers have studied the relation between spatial aptitude, measured by traditional test batteries, and spatial performance in the real world (Hegarty \& Kozhevnikov, 1999); however, there are no recommendations regarding spatial ability, its use, or its development in instruction.

\section{STRATEGIES AND INFORMATION PROCESSING RESEARCH}

A process-analytic approach has been used to examine quantitative differences in observed performance as well as qualitative differences with regard to the strategies used by individuals on spatial processing tasks (Bethell-Fox, Lohman, \& Snow, 1984; Cooper, 1975; Kyllonen, Lohman, \& Snow, 1984; Kyllonen, Lohman, \& Woltz, 1985; Lohman \& Kyllonen, 1983; Pellegrino \& Kail, 1981; Shepard \& Feng, 1972; Shepard \& Metzler, 1971; Sternberg \& Weil, 1980). Snow (1978) found that performance could be accounted for by three types of strategy differences: the sequence in which processing steps are executed, the steps taken to solve problems, or summation differences where the organization of processing can vary within and between persons as well as across items on a task.

Both aptitude and strategy variations have been described with regard to spatial visualization ability (Kyllonen et al., 1984; Kyllonen et al., 1985). Aptitude may limit strategy selection or predict performance within strategy subgroups or both. Conversely, strategy choice could be unrelated to aptitude, but the effectiveness of the implemented strategy could be limited by aptitude. Different individuals and skill groups have been shown to use alternative strategies for solving spatial problems. Some individuals compensated for deficits in visualization with verbal-analytic strategies and vice versa. Strategies for solving spatial items can shift from visual to verbal modes as a result of minor variations in tasks (Glushko \& Cooper, 1978; Hunt \& MacLeod, 1979; Sternberg \& Weil, 1980). Flexibility of strategy use, or strategy shifting, reflects an individual's adaptation to changes in task difficulty. Apparently, higher ability students can apply their knowledge of the stimulus and task constraints to reduce their processing burden by using their prior figural knowledge to reduce the complexity of the spatial task. Study 1 explores such performance differences on a real-world spatial task.

\section{STUDY 1: A COGNITIVE TASK ANALYSIS OF THE ORTHOGRAPHIC PROJECTION TASK}

A process-analytic approach is used to discover which skills are necessary to solve a particular spatial task known as orthographic projection. Orthographic projections are two-dimensional representations of three-dimensional objects (see Figure 1). Usually an object can be described by a set of orthographic projections that consist of the top, front, and right side front on views. This spatial task is an engineering design problem rather than a test created to measure aptitude. Individuals are asked to recognize or construct a three-dimensional object from a set of two-dimensional drawings.

Past research has shown that there are at least two effective strategies employed on spatial visualization tasks (Carpenter \& Just, 1986; Glushko \& Cooper, 1978; Hunt \& MacLeod, 1979; Kyllonen et al., 1984). One such strategy is a constructive approach in which individuals mentally construct visual representations of objects that require transformations necessary for problem solution. An analytic strategy consists of employing a sequential feature-by-feature method of comparison and transformation. Similar strategy differences have been found underlying performance on orthographic projection items (Cooper, 1983; Pellegrino et al., 1984). However, because a standardized test of orthographic projection items does not exist, it is difficult to compare research in this area. It is difficult to predict whether or not these same strategy differences will occur for orthographic projection problems with different test formats and what types of aptitude strategy in-
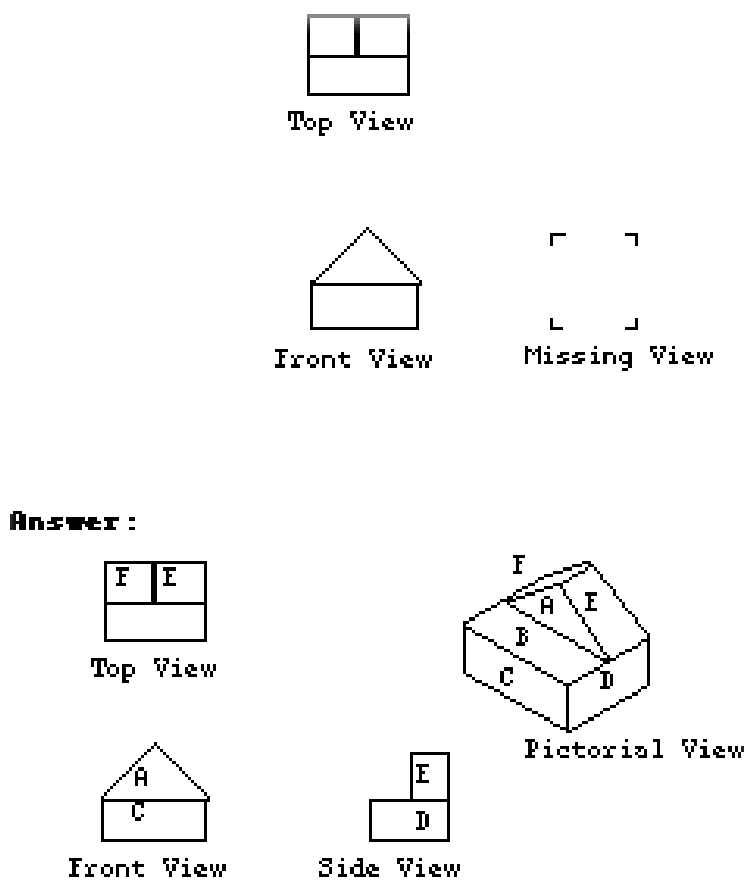

FIGURE 1 Sample orthographic projection item: Draw the missing side orthographic view. 
teractions might apply to them. This study expands on the existing knowledge of how individuals solve these problems. A cognitive task analysis was performed and verbal protocols were obtained from individuals while they attempted these problems. By asking experts and novices to solve these items, it was hoped that the nature of efficient strategies as well as common misconceptions that individuals encounter with this spatial task would emerge. Several research questions were studied: Because orthographic projections appear to require complex mental transformations, as do tests of spatial visualization, which spatial ability tasks are correlated with performance on the orthographic projection task? Are there alternative strategies for solving orthographic projections? Do relatively expert and novice visual thinkers differ in their strategies for solving orthographic projections?

\section{Method}

Participants. A total of 17 skilled and 16 less skilled participants were selected on the basis of their experience with orthographic projection problems. Most participants had graduate degrees and were in the high-ability range. Skilled individuals had taken at least one course in mechanical drawing, were familiar with orthographic projection problems, and were employed by companies where some knowledge of mechanical drawing was required. In the skilled group there were 14 engineers, 2 mechanical draftspersons, and 1 computer scientist who specialized in computer graphics. Individuals in the less skilled group had no prior courses or experience with orthographic projections and were involved in occupations that were not identified with spatial or visual thinking. Of the novices, 14 were educational psychology students, and 2 were from a humanities department. Attempts were made for a balanced sample; however, men tended to outnumber the women in the engineering sample (14 men and 3 women), and the reverse was true in the education sample (6 men and 10 women). Participation was voluntary.

Test descriptions. The test battery included measures of verbal, fluid, and spatial ability. The verbal and spatial aptitude tests were the advanced vocabulary V-4 (ADV) test, and the paper-folding (PF) and surface development (SD) tests, respectively (Ekstrom, French, Harman, \& Dermen, 1976). The letter series (LS) test (Thurstone, 1938) was used as a measure of fluid ability. Because a standardized orthographic projection test did not exist, the author designed a test based on items common in mechanical engineering textbooks, such as French \& Vierck (1978; see Figure 1).

The investigator administered tests individually. Testing took approximately $2 \mathrm{hr}$ per individual. Parallel forms of the orthographic projection test were administered. Part 1 was used to collect latency and accuracy data, and Part 2 was used to collect verbal protocols during problem solving.

\section{Results}

Characteristics of sample. Aptitude profiles were obtained from the performance scores. Group differences on the test battery were analyzed by performing $t$ tests on each of the performance scores. Experts performed significantly higher than the novices on the spatial tasks and orthographic projection test, whereas novices performed better than the experts on the verbal test (see Table 1). Both groups scored well on the measure of fluid ability (LS).

Correlational analysis. As expected, the spatial aptitude measures, surface development task $(r=.77, p<.05)$ and paper folding task $(r=.69, p<.05)$, had the highest correlation with the orthographic projection task, and no correlation was found between verbal aptitude and performance on the orthographic projection task $(r=-.17, p>.05)$.

Strategy differences. The expert sample had a 77\% accuracy rate on the orthographic projection task, whereas the novices had a score of $47 \%$ correct. An intensive analysis was conducted to study the strategic differences across groups on the orthographic projection test. A description of the coding scheme is presented in the Appendix. Introspections revealed that participants with $100 \%$ accuracy, whether in the expert or novice category, used a constructive strategy. The constructive strategy consisted of visualizing or drawing a three-dimensional model of the item in question before drawing the missing view. The constructive strategy consisted of several components: (a) feature decomposition; (b) rotating, folding, and orienting; (c) hypothesis generation; and (d) verifying or checking hypotheses.

Feature decomposition refers to examining the orthographic views feature by feature, hypothesizing about what a feature looks like in one view and testing the hypothesis in the next view. For instance, one might hypothesize that a feature represents a recessed plane and test that assumption in both the top and front views. By performing this decomposition systematically, it is possible for individuals to construct a three-dimensional image or model of what the item represents. In addition to feature decomposition, some people describe rotating the top, front, and side views together by mentally folding them into a model of the item. Other individuals draw a three-dimensional picture of the item. The constructive strategy allows them to put forth multiple hypotheses regarding what the item represents. This flexibility allows them to discard a hypothesis and start over again, resulting in a higher accuracy rate. A final element of this strategy is the verification process. Efficient individuals check and recheck their assumptions even when they have a correct hypothesis. Some individuals confirm their hypotheses by drawing a three-dimensional representation of their image before drawing the third view. Others obtain a three-di- 
TABLE 1

Expert-Novice Differences on the Test Battery

\begin{tabular}{|c|c|c|c|c|c|c|c|c|}
\hline & \multicolumn{2}{|c|}{ Sample Size } & \multicolumn{2}{|c|}{$M$} & \multicolumn{2}{|c|}{$S D$} & \multirow[b]{2}{*}{$t$ Value } & \multirow[b]{2}{*}{$d f$} \\
\hline & Novices & Experts & Novices & Experts & Novices & Experts & & \\
\hline Paper folding & 16 & 17 & 6.64 & 8.69 & 2.21 & 1.45 & $-3.18^{*}$ & 31 \\
\hline Surface development & 16 & 17 & 18.38 & 26.41 & 7.53 & 4.52 & $-3.69 *$ & 24 \\
\hline Advanced vocabulary & 16 & 17 & 14.91 & 12.14 & 2.45 & 3.63 & $2.56^{*}$ & 31 \\
\hline Letter series & 16 & 17 & 11.13 & 12.18 & 2.25 & 1.98 & -1.43 & 31 \\
\hline Orthographic projection test & 16 & 17 & 11.50 & 18.76 & 4.31 & 4.36 & $-4.81^{*}$ & 31 \\
\hline
\end{tabular}

$* p<.05$.

mensional image but verify their measurements of the side-view lines by examining the lines in the top and front views. Each stage of this strategy consists of forming an image or parts of an image and testing to see if it solves a particular problem.

Experts generally have larger memory banks for their particular domain. For this particular task, experts are able to generate multiple hypotheses for features in each view, and they are also able to systematically decompose a figure feature by feature. An expert might mention the position of the features, such as the front, back, and height of an object. These positioning cues may be a form of description, but they may also serve to assist individuals to orient their images. Checking mechanisms are also in place, as evidenced by statements such as "This line in the center disturbs me." Experts may recognize problems with their original assumptions and reformulate their subsequent hypothesis.

Less skilled participants used a mixture of constructive and analytic strategies. An analytic strategy in this case refers to matching lines in each view rather than visualizing an object. The analytics depend on line extensions to get their third view as opposed to decomposing features and imaging the three-dimensional object. Individuals who have difficulty with these problems use a combination of the analytic and constructive strategies but use them ineffectively. Participants will attempt to visualize an object but will not be systematic enough in visualizing each feature of the item. Even though they try to hypothesis test, they have a more limited repertoire of hypotheses regarding visual planes and what they might represent. These participants often do not verify their answers because they have not generated an answer.

An error analysis of the protocol data was conducted to identify the common mistakes or misconceptions individuals had with orthographic projection problems (see Lajoie, 1986). This analysis served to develop a diagnostic model of remediation used in OPT.

\section{Conclusion}

Both groups consisted of high-ability individuals as indicated by their scores on tests of fluid ability. Still, expert and novice visual thinkers differed both in aptitude profiles and in their performance on the orthographic projection test. The experts performed higher than the novices on the orthographic projection test and on tests of spatial ability, whereas the novices did better than the experts on the verbal aptitude measure. These results suggest that the experts and novices had definite aptitude strengths. Spatial ability was highly correlated to proficiency on the orthographic projections, whereas verbal ability tended to be negatively correlated to good performance on this task. Compensatory strategies may succeed in simpler spatial tasks, such as paper folding (Kyllonen et al., 1984), but not in difficult ones like the orthographic projection task.

The fact that psychometric tests of spatial aptitude were found to correlate highly with the orthographic projection test indicates that the orthographic projection test represents the same constructs as that of other spatial tasks. Cooper (1983) did not find a strong relation between spatial aptitude and performance on the orthographic projections. However, there were differences in the problem format, aptitude tests, and the samples used in the two studies that could account for the different results.

Experts in this research had a higher accuracy rate on the orthographic projection items than the novices. However, there were novices who had perfect scores on these items and performed as efficiently as the experts. Apparently, the most efficient participants used the same strategy (constructive) for solving these problems.

Individual differences in solving this task can be explained from an expertise perspective as well as a process perspective. Research on competence building suggests that experts tend to have more hierarchically based representations of knowledge (Chase \& Chi, 1981). Empirical evidence suggests that high-spatial-ability individuals have more highly organized mental representations of objects than low spatials (Lohman \& Nichols, 1985). The protocols in Study 1 revealed that experts had a greater repertoire of schema for twoand three-dimensional objects. Experts were able to generate a wider variety of visual hypotheses when looking at the two orthographic views. More experts than novices drew the three-dimensional object in an attempt to find the missing view. Novices tried to map the images to familiar items but had difficulty with complex items.

Evidence suggests that there were sequence, route, and summation differences (Snow, 1978) in the observed strate- 
gies. Individuals differed in the order in which they executed certain processing steps. For instance, some people would start with the top view, others the front view. Some individuals who used a constructive strategy rotated or folded the two views together before they synthesized these views into a three-dimensional image, whereas others decomposed specific features and constructed images of these features before they folded the entire image into the proper representation. Other individuals did these steps in the exact opposite manner. Evidence of route differences, or differences in the steps people took to solve the problems, were apparent in that there were constructive and analytic strategies and differences within each strategy. Strategy shifting between the constructive and analytic strategies was most prevalent in the novice sample. These novices seemed to be trying the best of both strategies in an attempt to discover the answer. Aptitude may limit strategy selection or predict performance or both in strategy subgroups (Bethell-Fox et al., 1984; Kyllonen et al., 1984; Kyllonen et al., 1985).

The cognitive task analysis provided detailed information on how experts and novices solved problems in this domain. This information was used to develop a computerized tutor with a built-in diagnostic capability for providing individualized feedback on strategy usage and ways to remediate the particular bugs or misconceptions identified in the cognitive task analysis. OPT is described later in this article as a computer tutor that scaffolds learners by teaching lower order skills first followed by more complex skills later.

\section{STUDY 2: CAN SPATIAL APTITUDE BE TAUGHT?}

Study 2 was conducted to establish whether or not a computer-based learning environment, OPT, could enhance performance on a real-world spatial task.

\section{$\mathrm{OPT}^{1}$ : The Tutor Design}

An important aspect of tutor building is structuring the curriculum layer of a tutor in a way that builds on a learner's prior knowledge (Lesgold, 1986). One theory of skill acquisition is that knowledge is acquired in stages where knowing "what something is" or factual knowledge generally precedes knowing "how to do something" or procedural knowledge (Anderson, 1993). Establishing a learning hierarchy, whereby prerequisite skills are tutored prior to higher order knowledge, can ensure that knowledge is acquired systematically (Gagné, 1962). OPT was designed with this assumption, using a "bite-size architecture" (Bonar, 1985), where the ex-

\footnotetext{
${ }^{1}$ See Lajoie, 1986, for a full description of the tutor. The tutor was implemented on the Xerox 1109 and written in LISP.
}

pert system paradigm is used to establish the sequence of "mind-sized" bites of knowledge to the novice learner. Each bite represents a manageable chunk of knowledge. In OPT, the learning hierarchy consists of six tutoring sessions or bites, half that represent declarative and half that represent procedural knowledge. Declarative knowledge refers to what students need to know to help them solve orthographic projections. Sessions that facilitated line and shape comprehension as well as rules of symmetry were presented. The procedural knowledge bites are designed to teach individuals how to apply their knowledge in problem-solving contexts. These sessions involved surface recognition or development, drawing orthographic projections, and hypothesizing about what the three-dimensional views would look like based on sets of orthographic views. A detailed description of the instructional issues that are relevant to the individual tutor bites is provided in the following sections.

Declarative knowledge: What is to be learned? Based on the assumption that spatial aptitude can be trained, it is likely that individuals have a mental model of spatial concepts that can be refined and modified (Lohman \& Nichols, 1985). For some individuals, building a spatial lexicon is comparable to learning a foreign language. A vocabulary has to be learned before a sentence can be understood or stories generated. Likewise building a mental model of spatial representations, a figural vocabulary must be learned before complex spatial reasoning can occur. For this reason, OPT tutors individuals about how line, shape, and symmetry are represented in two and three dimensions.

The line-comprehension section consists of several sub-bites of information, those being vertical, horizontal, diagonal, and hidden lines. Examples of these individual line segments and how they are represented in orthographic and isometric projections are provided to the learner. An example of a vertical line embedded in the top, front, and side orthographic views will be presented with a corresponding isometric view. Such examples extend the comprehension of what a line looks like in one orientation versus another.

The line-comprehension demonstration presents an isometric view with three orthographic views. The line in question is highlighted in the orthographic view, and the isometric rotates to the orthographic view in question (top, front, or side) and highlights the line that individuals should translate. Similar demonstrations occur in the shape-comprehension and symmetry bites, only specific shapes are highlighted and identified instead of lines. Figure 2 provides an example of how the shape of a square on the front orthographic view would translate in the isometric three-dimensional view and in the side view.

Procedural knowledge: How to use the knowledge in a problem-solving context. The procedural knowledge section provides people with monitoring techniques as well as analytic and constructive strategies for solving ortho- 

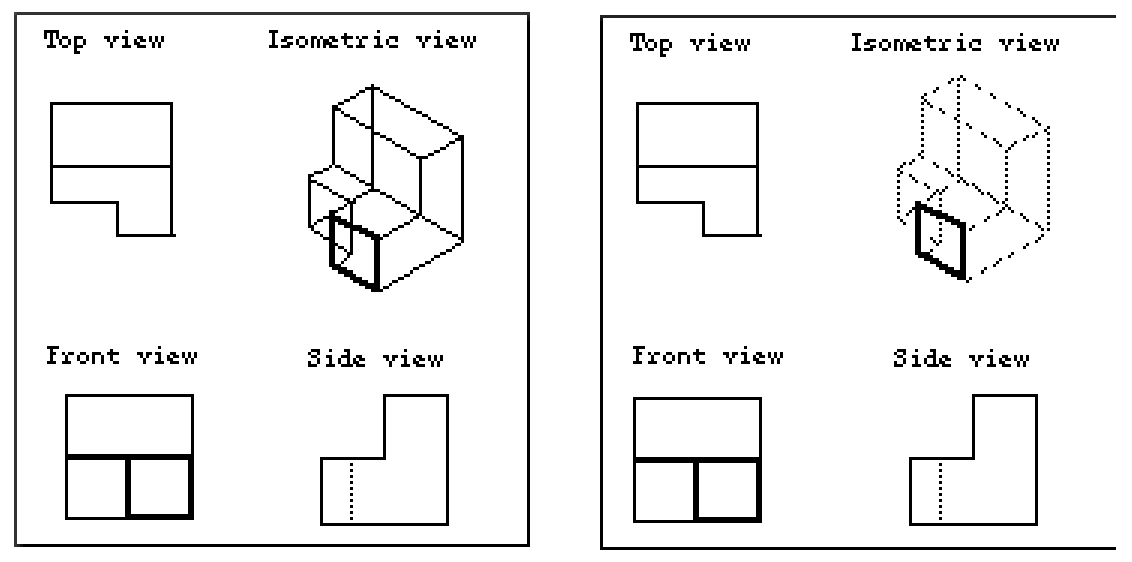

FIGURE 2 Tutoring shape translation in OPT.

graphic projection problems. While solving problems in this section, they can review the lessons they learned in the spatial lexicon at any point to help them with their problem solving. The procedural section tutors students in surface recognition, drawing, and hypothesizing.

The surface recognition bite teaches individuals metacognitive strategies for checking their own performance. For each surface in the two-dimensional representations there is a corresponding one in the isometric view. Students are asked to count or match surfaces from one view to another. If their solution is incorrect, the isometric view will rotate to the view in question so that the student can see how many surfaces are present and which surfaces match their drawings. In the error identification bite, individuals must identify and correct errors using a drawing tool kit to remove and add lines to the orthographic view.

In the draw-bite section, individuals construct rather than recognize a correct answer. Their task is to draw one of three orthographic projections of a given isometric view. A playback of the student's answer is provided, which demonstrates what part of the answer is correct and where changes need to occur. Remediation is presented based on the type of line error.

In the final section of this tutor, hypothesize bite, individuals must construct an orthographic projection without the aid of an isometric. There is a set of sub-bites leading up to this goal, which range from recognition of correct drawings to constructing the correct missing view.

The tutor presents demonstrations of the rotation or visualization strategy more directly than the analytic strategy, but both are implicit within the tutor. The constructive strategy builds on what has been shown earlier in the draw bites. It combines feature decomposition and rotation with a multiple-hypothesis-generation philosophy where individuals are encouraged to form multiple three-dimensional hypotheses by generating images for both of the orthographic projections and then synthesizing these two views into one picture. The analytic method is tutored by helping students translate lines from the orthographic views to an isometric image as well as mapping lines from the top and front orthographic views to the side view.

The computer monitors the performance in each session, generates a model of what the student knows, and subsequently generates a problem at the right level of difficulty for that individual. There is a diagnostician and an expert for each bite, which determines whether a student has reached mastery or not and what remediation, if any, is necessary for that individual. Hence, moving up the bite hierarchy parallels the learning hierarchy from lower level factual knowledge to higher order skills. Remediation is presented in the context of the problem. Each session is designed to present specific knowledge that is prerequisite to the subsequent bites in the hierarchy.

\section{Participants}

Thirty undergraduate students from University of Pittsburgh were recruited and paid to participate. Students were selected if they had no experience in mechanical drawing and their major was in a field that did not emphasize spatial skills. Students were randomly assigned to one of three groups, Control Group 1, Control Group 2, or the treatment group, with 10 per group; there was not a large enough pool of students to allow for an equal number of men and women in each group. The gender breakdown for the three groups was as follows: (a) in Control Group 1, there were 2 men and 8 women; (b) in Control Group 2, there were 4 men and 6 women; and (c) in the treatment group, there were 3 men and 7 women.

\section{Experimental Groups}

Individuals were tested prior to and after treatment on tests of orthographic projections to measure change in performance. Two control groups were used to ensure that any learning observed for the treatment group was not due simply to practice, 
or practice with feedback regarding correctness of solutions. Thus, Control Group 1 was given a pre- and posttest of orthographic projections, whereas Control Group 2 was given the pretest with an answer sheet. Students in the latter group were told to check their answers with the solutions provided on the answer sheet. The tutor instruction was self-paced, and all participants were tested individually. On average the session times varied between 50 and $90 \mathrm{~min}$.

\section{Test Materials and Administration}

The same aptitude measures as Study 1 were used with the addition of the Raven Advanced Matrices test, a measure of fluid ability. Two equivalent sets of items were established for the pre- and postorthographic projection tests (see Lajoie, 1986). Testing occurred in a 3 -week period. The orthographic projection pretests as well as the aptitude measures were administered to everyone in Week 1 . The treatment group received the tutor intervention in Week 2, whereas the control groups did not receive instruction. In Week 3 all participants received the orthographic projection posttest.

\section{Results}

The results section is divided into three parts. The first section provides information on the participant's aptitudes. Part 2 presents the group differences information for pre- and posttests. The tutor data is described in terms of reaction times and accuracy in Part 3.

Aptitude profiles. Figure 3 illustrates the aptitude profiles for all three groups. On average, all groups performed highest on tests of fluid intelligence, the letter series (80\%) and Ravens (68\%), followed by tests of spatial ability, paper folding $(60 \%)$, and the orthographic pretest $(55 \%)$. They tested poorly on verbal ability, advanced vocabulary (53\%).

A multivariate analysis of variance (MANOVA) was conducted, which examined raw score group differences on the entire test battery to check whether or not there were group differences on the tests as a whole. This was done by examining the multivariate $F$ test; on individual tests the univariate $F$ was examined. There were no significant group differences on the test battery, $F(14,42)=.88 p>.05$, and hence are not reported. There was a trend for the treatment group to score lower than the other groups on pretests, in general, but not significantly.

In Table 2, descriptive data are provided on gender differences for the entire test battery. Percentages are presented in this table for comparison purposes across measures; however, the analysis was conducted on raw scores. Significant gender differences were found for men scoring higher than women on the advanced vocabulary and orthographic preand posttests. However, the men tended to score higher than women on the entire test battery.

Correlational analyses. A correlational analysis was conducted to understand what aptitude measures were associated with accuracy on the orthographic projection test. An analysis of the total sample as well as the three separate groups was performed to see whether the correlational structure was the same for all three groups and to elaborate on how they were the same or different. There was a high correlation between the orthographic pre- and posttest for the total sample, Control Group 1, Control Group 2, and the treatment group (because patterns were similar for each group, only the total sample is reported in Table 3). In addition, the orthographic tests correlated higher with themselves than with any other tests.

The sample's performance on the test battery indicated that all tests were significantly intercorrelated except for the advanced vocabulary test, which was only correlated with the orthographic pretest. However, when examining the three groups independently, this verbal test was not correlated with performance on any other test. The orthographic projection tasks were intercorrelated with tests of fluid and spatial ability.

Performance differences on the orthographic projection tests. A repeated measures analysis of variance (ANOVA) tested whether or not performance on the or-

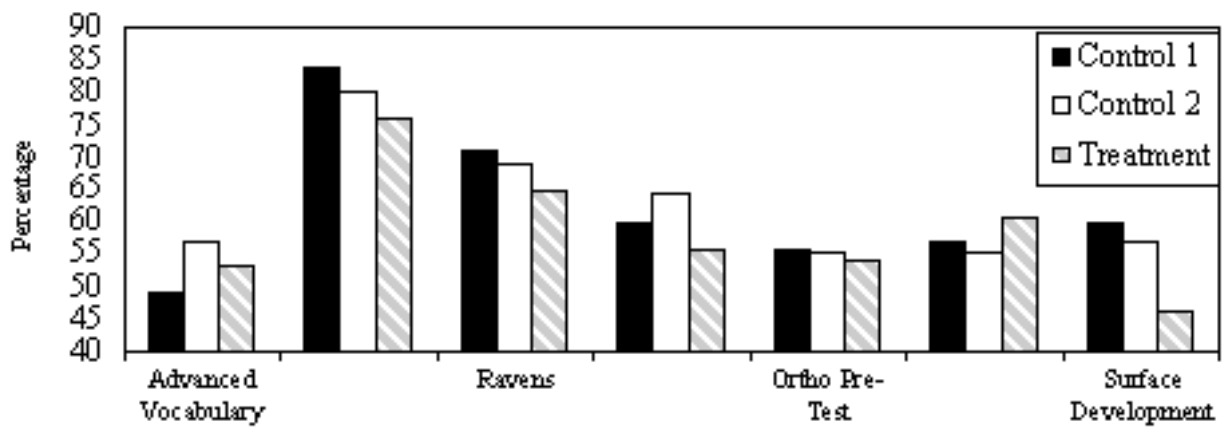

FIGURE 3 Aptitude Profiles for 3 Experimental Groups. 
TABLE 2

Test Battery Breakdown by Gender

\begin{tabular}{|c|c|c|c|c|c|}
\hline \multirow[b]{2}{*}{ Test } & \multicolumn{2}{|c|}{ Men } & \multicolumn{2}{|c|}{ Women } & \multirow[b]{2}{*}{$F(d, f)$} \\
\hline & $\%$ Accuracy & $S D$ & $\%$ Accuracy & $S D$ & \\
\hline Advanced vocabulary & 65 & 12.6 & 48 & 11.7 & $11.10(1,27)^{*}$ \\
\hline Letter series & 84 & 13.1 & 79 & 15.9 & $0.63(1,27)$ \\
\hline Ravens & 72 & 17.0 & 67 & 13.6 & $0.90(1,28)$ \\
\hline Paper folding & 68 & 16.6 & 57 & 16.6 & $2.90(1,28)$ \\
\hline Orthographic projections (pre) & 74 & 17.2 & 47 & 19.1 & $12.80(1,28)^{*}$ \\
\hline Orthographic projections (post) & 70 & 15.5 & 54 & 13.7 & $7.40(1,28)^{*}$ \\
\hline
\end{tabular}

TABLE 3

Test Battery Intercorrelations for Total Sample

\begin{tabular}{|c|c|c|c|c|c|c|}
\hline & $A D V$ & $L S$ & $R A V$ & $P R E$ & POST & SURFACE DEV \\
\hline Paper folding (PF) & .10 & .35 & .56 & .54 & .41 & .58 \\
\hline Advanced vocabulary (ADV) & - & .16 & -.05 & .31 & .24 & .23 \\
\hline Letter series (LS) & - & - & .57 & .65 & .64 & .68 \\
\hline Raven progressive matrices (RAV) & - & - & - & .59 & .65 & .71 \\
\hline Orthographic projection pre (PRE) & - & - & - & - & .88 & .76 \\
\hline Orthographic projection post (POST) & - & - & - & - & - & .71 \\
\hline
\end{tabular}

Note. $n=30 ; r=.35 ; p<.05$. SURFACE DEV = surface development task

thographic projection task improved with practice, practice with feedback, or with tutoring. A Group (treatment and Control Groups 1 and 2) $\times$ Gender (2) $\times$ Test (pre- and postorthographic test) $\times$ Problem type (1-4) analysis was performed and the results are shown in Table 4. The test and problem type variables were the repeated measures.

There were no significant between-group differences in pre- and posttests $F(2,24)=.4, p>.05)$. However, there was a significant main effect of gender, $F(1,24)=10, p<$ .05 , and problem type, $F(3,72)=47, p<.05$. Interactions were also found. There was a significant interaction between test and gender, $F(1,24)=7.5, p<.05$, problem type and gender, $F(3,72)=5.8, p<.05$, and test by problem type, $F(3,72)=7.7, p<.05$.

The men performed better on both the pre- $(M=75)$ and posttests $(M=70)$ than women (pretest $M=45$ and posttest $M=$ $55)$ but more so on the pre- than on the posttest. The men declined in performance from pre- to posttest, whereas the women increased their scores. This effect may be due to the treatment or simply a regression of these groups to the mean performance score. The problem types were designed so that Types 2, 3, and 4 are more difficult than Problem Type 1, in that order. However, the test by problem type interaction indicates that in the pretests Problem Type 1 was more difficult than Problem Type 2, whereas the reverse was true in the posttest. Individuals tended to do better on Problem Types 1 and 3 in the posttests than in the pretests and did worse in the other types. There was also a gender-by-problem-type interaction. Men tended to do better than women on all problem types. Apparently, men found Types 1 to 3 fairly simple, averaging around $78 \%$ accuracy, whereas they only scored $55 \%$ correct on Problem Type 4. On the other hand, women indicated a steadier decline in performance from Problem Type 1 to 4, finding Type 1 the simplest and Type 4 the most complex. The women performed in the expected direction because the problem types were designed to reflect a gradient of difficulty, Type 1 being the simplest and Type 4 the most complex.

Although between-group differences were not found on the pre- and posttests, a within-group analysis of performance over time on the pre- and posttests was revealing. The use of $t$-test pairs on the three separate groups for pre- and posttests revealed that the treatment groups had a tendency to score higher on the posttest than the control groups (see Table 5). This led to a scrutiny of the raw data in the form of scatter plots for the separate groups on these tests. The scatter plots showed that each group had outliers. By ignoring those individuals who scored extremely well on the orthographic pretests (1 $S D$ above the mean), the performance gains due to the tutor interaction were more substantial. Individual aptitude profiles were developed for each participant in the tutor sample. These data indicate that 7 out of the 10 participants had a gain in performance from the pre- to the postorthographic test. Those who did not show performance gains were of high spatial ability. Perhaps specific tutoring on OPT interferes with their already efficient spatial strategies.

\section{Aptitude and Gender Differences}

Although there were no significant differences between the control groups and the treatment group on the orthographic 
posttest, there were significant performance gains for the treatment group, and only this group demonstrated significant gains from pre- to posttest. This result is significant considering that this group tended to have lower pretest scores than the control participants and still resulted in higher, if not significantly higher, posttest scores than the other two groups.

The lower aptitude individuals benefited more from training than did the higher aptitude individuals. There is evidence in aptitude research to support the fact that low-ability participants gain more from training then do high-ability individuals. Examining the characteristics of the tutor sample indicated that those individuals with high spatial aptitude decreased their performance on the posttest. Perhaps the tutor interaction interfered with their already efficient processes. This speculation would support the contention that high-ability individuals may decrease their performance scores with training due to an interference of training with already existing strategies (Cronbach \& Snow, 1977; Salomon, 1974).

This study was not designed to examine gender differences. However, the results suggest that training on OPT improves performance on the orthographic projection task for women more than men. On first observation, this finding supports the literature indicating that men do better on tests of spatial aptitude than women (Maccoby \& Jacklin, 1974). On the other hand, aptitude differences were confounded with these gender differences. The men in this study tended to do better on all of the aptitude measures than did the women but only significantly better on the orthographic tests and the advanced vocabulary test. The sample size in this research was too small to conclude that only women will benefit by training on OPT. However, the results do suggest that those individuals with low spatial aptitude will benefit from tutoring.

\section{Nature of the Treatment: Implications for Instruction}

The cognitive task analysis of skilled and less skilled performance on the orthographic projection task led to a description of the rich body of strategic knowledge that skilled performers use to solve these problems. The less skilled individuals provided valuable information on the types and frequencies of errors encountered. Thus, the cognitive task analysis

TABLE 4

Repeated Measures Analysis of Variance: Group (3) $\times$ Gender (2) × Test (2) $\times$ Problem Type (4)

\begin{tabular}{|c|c|c|c|}
\hline Source & $d f$ & $M S$ & $F$ \\
\hline Group (Grp) & 2 & 9.9 & 0.4 \\
\hline Gender (Gnd) & 1 & 271.8 & $10.0^{*}$ \\
\hline Grp $\times$ Gnd & 2 & 53.0 & 2.0 \\
\hline Error & 24 & 27.2 & \\
\hline Test & 1 & 0.5 & 0.2 \\
\hline Test $\times$ Grp & 2 & 2.1 & 0.7 \\
\hline Test $\times$ Gnd & 1 & 21.8 & $7.5^{*}$ \\
\hline Test $\times$ Grp $\times$ Gnd & 2 & 1.3 & 0.4 \\
\hline Error & 24 & 2.9 & \\
\hline Problem type (PT) & 3 & 194.5 & $47.0 *$ \\
\hline PT $\times$ Grp & 6 & 1.8 & 0.5 \\
\hline $\mathrm{PT} \times$ Gnd & 3 & 24.0 & $5.8^{*}$ \\
\hline $\mathrm{PT} \times \mathrm{Grp} \times \mathrm{Gnd}$ & 6 & 3.5 & 0.8 \\
\hline Error & 72 & 4.1 & \\
\hline Test $\times$ PT & 3 & 19.9 & $7.7 *$ \\
\hline Test $\times$ PT $\times$ Grp & 6 & 1.9 & 0.7 \\
\hline Test $\times$ PT $\times$ Gnd & 3 & 3.8 & 1.5 \\
\hline Test $\times$ PT $\times$ Grp $\times$ Gnd & 6 & 1.9 & 0.7 \\
\hline Error & 72 & 2.6 & \\
\hline
\end{tabular}

${ }^{*} p<.05$.

TABLE 5

Group Differences on Orthographic Pre- and Posttests

\begin{tabular}{|c|c|c|c|c|c|c|}
\hline & \multicolumn{2}{|c|}{ Control Group 1} & \multicolumn{2}{|c|}{ Control Group 2} & \multicolumn{2}{|c|}{ Treatment } \\
\hline & Pretest & Posttest & Pretest & Posttest & Pretest & Posttest \\
\hline$M$ & 27.40 & 28.5 & 26.50 & 26.5 & 25.80 & 29.7 \\
\hline$S D$ & 8.00 & 7.1 & 11.80 & 9.0 & 12.50 & 6.8 \\
\hline$t$ value $(d f=9)$ & -0.73 & & -0.02 & & $-2.10^{*}$ & \\
\hline
\end{tabular}

$* p<.07$ 
served as the basis for the diagnostic instruction in OPT. It provided information for a guided modeling approach for the tutor as well as links to the types of intelligent remediation that would be necessary at different phases of the tutor. The empirical work provided an understanding of the cognitive skills necessary to solve these tasks, which consequently led to the structuring of the tutor in a way that would provide the conceptual glue that would help students build on familiar knowledge in the context of a problem.

By examining the monitoring and checking mechanisms that the skilled individuals used in solving orthographic projection items, a curriculum was developed that taught individuals to use these mechanisms. Students had the opportunity to see why their answers were correct or incorrect.

OPT was developed to test the assumption that mental models of spatial representation could be altered through instruction. The computer served as a medium for extending learning and in fact supports the notion that computers can serve as cognitive tools that help students during thinking or problem solving (Jonassen \& Reeves, 1996; Lajoie, 2000; Lajoie \& Derry, 1993; Pea, 1985; Salomon, Perkins, \& Globerson, 1991). The aptitude process theories add to our understanding of how technology can assist learning, for whom, and how it can be designed based on cognitive task analysis methods and aptitude measures. There are still many avenues to explore before we fully understand how to design adaptive forms of instruction that are based on dynamic forms of assessment.

\section{ACKNOWLEDGMENTS}

This article draws on a dissertation completed by Susanne P. Lajoie under the guidance of Richard E. Snow at Stanford University. Lajoie won the Division 15 Dissertation Award from the American Psychological Association as well as an Honorable Mention for Best Dissertation at the American Educational Research Association in 1987. Although her advisor encouraged her to publish this research it was not until his untimely death that she was compelled to honor his wish. The dissertation research was funded by the Office of Naval Research, Maxima, and Universal Energy Systems Air Force contracts.

I gratefully acknowledge the assistance of Marguerite Roy in her careful review of this article and Marty Kent for his programming skills.

\section{REFERENCES}

Anderson, J. R. (1993). Rules of the mind. Hillsdale, NJ: Lawrence Erlbaum Associates, Inc.

Bethell-Fox, C. E., Lohman, D. F., \& Snow, R. E. (1984). Adaptive reasoning: Componential and eye movement analysis of geometric analogy performance. Intelligence, 8, 205-238.

Bonar, J. (1985). Bite sized intelligent tutoring (Tech. Rep.). Pittsburgh, PA: University of Pittsburgh, Learning Research and Development Center.

Carpenter, P. A., \& Just, M. A. (1986). Spatial ability: An information processing approach to psychometrics. In R. Sternberg (Ed.), Advances in the psychology of human intelligence (Vol. 3). Hillsdale, NJ: Lawrence Erlbaum Associates, Inc.

Chase, W., \& Chi, M. (1981). Cognitive skill: Implications for spatial skill in large scale environments. In J. Harvey (Ed.), Cognition, social behavior, and the environment. Potomac, MD: Lawrence Erlbaum Associates, Inc.

Cooper, L. A. (1975). Mental transformations of random two-dimensional shapes. Cognitive Psychology, 7, 20-43.

Cooper, L. A. (1983, April). Spatial information processing: The nature of strategic variation. Paper presented at the annual meeting of the American Educational Research Association, Montreal, Quebec, Canada.

Corno, L., Cronbach, L. J., Kupermintz, H., Lohman, D. F., Mandinach, E. B., Porteus, A. W., et al. (2002). Remaking the concept of aptitude: Extending the legacy of Richard E. Snow. Mahwah, NJ: Lawrence Erlbaum Associates, Inc.

Cronbach, L. J. (1970). Essentials of psychological testing (3rd ed.). New York: Harper \& Row.

Cronbach, L. J., \& Snow, R. E. (1977). Aptitudes and instructional methods: A handbook for research on interactions. New York: Irvington.

Ekstrom, R. B., French, J. W., Harman, H. H., \& Dermen, D. D. (1976). Manual for kit of factor referenced cognitive tests. Princeton, NJ: Educational Testing Service.

French, T. E., \& Vierck, C. J. (1978). Engineering drawing and graphic technology (12th ed.). New York: McGraw-Hill.

Gagné, R. (1962). The acquisition of knowledge. Psychological Review, 69, $355-365$.

Glushko, R. J., \& Cooper, L. A. (1978). Spatial comprehension comparison processes in verification tasks. Cognitive Psychology, 10, 391-421.

Hegarty, M., \& Kozhevnikov, M. (1999). Spatial abilities, working memory and mechanical reasoning. In J. Gero \& B. Tversky (Eds.). Visual and Spatial Reasoning in Design. Sydney, Australia: Key Center for Design and Cognition.

Hunt, E., \& MacLeod, C. M. (1979). The sentence-verification paradigm: A case study for individual differences. In R. J. Sternberg \& D. K. Detterman (Eds.), Human intelligence: Perspectives on its theory and measurement. Norwood, NJ: Ablex.

Jonassen, D. H., \& Reeves, T. C. (1996). Learning with technology: Using computers as cognitive tools. In D. H. Jonassen (Ed.), Handbook of research for educational communications and technology (pp. 693-719). NY: Macmillan.

Kyllonen, P. C., Lohman, D. F., \& Snow, R. E. (1984). Effects of aptitudes, strategy training, and task facets on spatial task performance. Journal of Educational Psychology, 76, 130-145.

Kyllonen, P. C., Lohman, D. F., \& Woltz, D. (1985). Componential modeling of alternative strategies for performing spatial tasks. Journal of Educational Psychology, 76, 1325-1345.

Lajoie, S. P. (1986). Individual differences in spatial ability: A computerized tutor for orthographic projection tasks. Unpublished doctoral dissertation, Stanford University, CA.

Lajoie, S. P. (Ed.). (2000). Computers as cognitive tools: Vol. 2. No more walls: Theory change, paradigm shifts and their influence on the use of computers for instructional purposes. Mahwah, NJ: Lawrence Erlbaum Associates, Inc.

Lajoie, S. P., \& Derry, S. J. (Eds.). (1993). Computers as cognitive tools. Hillsdale, NJ: Lawrence Erlbaum Associates, Inc.

Lesgold, A. (1986). Toward a theory of curriculum for use in designing intelligent tutoring systems. In H. Mandle \& A. Lesgold (Eds.), Learning issues for intelligent tutoring systems. New York: Springer-Verlag.

Lohman, D. F. (1979). Spatial ability: A review and reanalysis of the correlational literature (Tech. Rep. No. 8). Stanford University School of Education, Aptitude Research Project, CA.

Lohman, D. F., \& Kyllonen, P. C. (1983). Individual differences in solution strategy on spatial tasks. In R. F. Dillon, \& R. R. Schmeck (Eds.), Individual differences in cognition: Vol. 1. New York: Academic.

Lohman, D. F., \& Nichols, P. D. (1985, March). Spatial ability: The effects of encoding processes and representational quality on mental synthesis. 
Paper presented at the meeting of the American Educational Research Association, Chicago, IL.

Maccoby, E. E., \& Jacklin, C. N. (1974). The psychology of sex differences. Stanford, CA: Stanford University Press.

McKim, R. H. (1980). Experiences in visual thinking (2nd ed.). Monterey, CA: Brooks/Cole.

Pea, R. D. (1985). Beyond amplification: Using the computer to reorganize mental functioning. Educational Psychologist, 20, 167-182.

Pellegrino, J. W., \& Kail, R. V. (1981). Process analyses of spatial aptitude. In R. J. Sternberg (Ed.), Advances in the psychology of human intelligence: Vol. 1. Hillsdale, NJ: Lawrence Erlbaum Associates, Inc.

Pellegrino, J. W., Mumaw, R. J., \& Shute, V. J. (1984). Analyses of spatial aptitude and expertise. In S. Embretson (Ed.), Test design: Contributions from psychology, education and psychometrics. New York: Academic.

Salomon, G. (1974). Internalization of filmic operations in relation to individual differences. Journal of Educational Psychology, 66, 499-511.

Salomon, G., Perkins, D. N., \& Globerson, T. (1991). Partners in cognition: Extending human intelligence with intelligent technologies. Educational Researcher, 20, 10-16.

Shepard, R. N., \& Feng, C. (1972). A chronometric study of mental paper folding. Cognitive Psychology, 3, 228-243.

Shepard, R. N., \& Metzler, J. (1971). Mental rotation of three-dimensional objects. Science, 171, 701-703.

Smith, I. (1964). Spatial ability: Its educational and social significance. London: University of London Press.

Snow, R. E. (1977). Research on aptitudes: A progress report. In L. S. Shulman (Ed.), Review of research in education: Vol. 4. Itasca, IL: Peacock.

Snow, R. E. (1978). Theory and method for research on aptitude processes. Intelligence, 2, 225-278.

Snow, R. E. (1980). Aptitude processes. In R. E. Snow, P. A. Federico, \& W. E. Montague (Eds.), Aptitude, learning and instruction: Vol. 1. Hillsdale, NJ: Lawrence Erlbaum Associates, Inc.

Snow, R. E. (1989). Toward assessment of cognitive and conative structures in learning. Educational Researcher, 18(9), 8-14.

Sternberg, R. J., \& Weil, E. M. (1980). An aptitude X strategy interaction in linear syllogistic reasoning. Journal of Educational Psychology, 72, 226-239.

Thurstone, L. L. (1938). Primary mental abilities. Chicago: University of Chicago Press.

\section{APPENDIX: SCORING CRITERIA}

Individuals were given the top and front orthographic views of a three-dimensional object and were asked to construct the third orthographic right-side view, without being provided with a three-dimensional picture of said object. The two major strategies were constructive and analytic or some combination of the two. To be categorized into one of the two main strategies, a protocol had to embody the general definition of the strategy as well as contain at least 2 out of the 3 component processes.

\section{Strategy Definitions and Scoring Key}

\section{Constructive (CONST)}

A constructive strategy consists of forming a three-dimensional model of the particular item in question by using the two orthographic views that are provided. A great deal of this strategy depends on the ability to synthesize the two orthographic views into one three-dimensional representation. This strategy consists of the following components:

Feature decomposition (FD). This consists of looking at each of the orthographic views feature by feature. Individuals decompose the two-dimensional views into component parts as a way to formulate hypotheses as to what these parts would look like in three dimensions. Once individuals hypothesize about what a feature looks like in one view, they test this hypothesis in the next view. If their hypothesis makes sense in both the top and front views, they carry on to the next feature represented in the item and speculate what it looks like. If their hypotheses are not consistent in the two views, they discard them and generate new hypotheses. By performing this feature decomposition systematically, it is possible for individuals to construct a three-dimensional model of the item.

Rotation, folding, and orientation (RFO). Some people describe rotating the top, front, and side views together by mentally folding them together as if they were on hinges. For instance, the top falls backward over the front view and the front and top then join the side view by bending the side view back. Some individuals describe orienting themselves to the side of the object so they can draw that view.

Verify and check (VER). This refers to checking their answers in terms of the hypotheses generated and to the scaling of their answers-in other words, are their measurements correct? Some individuals draw a three-dimensional picture to check their side orthographic view. Others use the other orthographic views to test their line placement.

\section{Analytic (ANAL)}

Consists of analyzing the orthographic views in terms of lines instead of three-dimensional shapes. This strategy does not consist of imagining a three-dimensional object or visualizing the object. It consists of the following components:

Line matching $(L M)$. Matching lines from the top to the front view to see how they fit together.

Line extension (LE). Extending lines from the top to the front, from the top to the side, and from the front to the side to draw the side orthographic view.

Verification (V). Individuals check to see that all lines are accounted for.

Constructive/analytic $(C / A)$. This strategy combines the two strategies by switching back and forth between the two in attempting to solve an item. 